\title{
The Electrical Parameters Modeling and Experimentation of Copper Vapor Laser
}

\author{
Fatemeh Rafighi' ${ }^{1}$, Saeed Behrouzinia ${ }^{2 *}$, Kamran Khorasani², Masoud Sabaghi², \\ Saeid Marjani ${ }^{3}$ \\ ${ }^{1}$ Central Tehran Department of Physics, Islamic Azad University, Tehran, Iran \\ ${ }^{2}$ Laser and Optics Research School, Nuclear Science and Technology Research School, Atomic Energy \\ Organization of Iran, Tehran, Iran \\ ${ }^{3}$ Department of Electrical Engineering, Ferdowsi University of Mashhad, Mashhad, Iran \\ Email: "sbehrouzi@aeoi.org.ir
}

Received 29 November 2015; accepted 10 January 2016; published 13 January 2016

Copyright (C) 2016 by authors and Scientific Research Publishing Inc.

This work is licensed under the Creative Commons Attribution International License (CC BY).

http://creativecommons.org/licenses/by/4.0/

(c) (i) Open Access

\begin{abstract}
In this paper, the present developments of copper vapor laser technology were computational modeling and simulations methods. In most of the cases, before the experiment, the functions of electrical parameters can be predicted by the use of physical simulation codes. A single simulation model describing the discharge circuitry was introduced. By solving the equations of circuit via Runge-Kutta method, the current and voltage pulses of the discharge tube were investigated, which are in good agreement with the experimental results of the same laser.
\end{abstract}

\section{Keywords}

\section{Copper Vapor Laser, Electrical Parameters, Simulation Method}

\section{Introduction}

In recent decade, the characteristics of lasers have improved extremely [1]-[25].The copper vapor lasers (CVL) with green and yellow wavelengths of $510.6 \mathrm{~nm}$ and $578.2 \mathrm{~nm}$ are known for their high average output power (higher than $100 \mathrm{~W})$, high repetition frequencies (1 - $150 \mathrm{kHz}$ ) and high efficiency (1\% - 2\%) [26] [27].

At present, CVLs are used as pumping sources for frequency conversion to visible, ultra violet and infra-red ranges, conducting lasers, skin diseases treatment and oncology. It is also increasingly applied in industries and material processing [28]. Computational modeling and simulation methods have been used for microscopic understandings of electric discharge pulse and presentation of more information on plasma kinetics, and have pro-

\footnotetext{
${ }^{*}$ Corresponding author.
}

How to cite this paper: Rafighi, F., Behrouzinia, S., Khorasani, K., Sabaghi, M. and Marjani, S. (2016) The Electrical Parameters Modeling and Experimentation of Copper Vapor Laser. Circuits and Systems, 7, 23-28.

http://dx.doi.org/10.4236/cs.2016.71003 
vided the details of kinetic processes in plasma discharge field, where the experimental methods are not applicable [29] [30]. Since some parameters of the lasers such as excitation rate and relaxation of the excited levels can't be directly measured, the modeling methods become more important. In most of the cases, before the experiment, the functions of some of the electrical parameters can be predicted by the use of physical simulation codes. Also before the laser devices are practically developed, simulation methods can predict that how the laser function is dependent on the external exciting parameters and geometrical characteristics of the circuit. In this work, at first, the differential equations of circuit function in a CVL were obtained, and then the simulations were carried out via solving of equations by Runge-Kutta method. The presented simulation method describes the current-voltage pulses behavior of a CVL with diameter of $11 \mathrm{~mm}$ and length of $580 \mathrm{~mm}$. by using of initial data from experimental tests carried out on the same laser and by comparing the results obtained from two experimental and simulation data, a good agreement was observed.

The paper is organized as follows: Section 2 briefly describes the discharge circuit characteristics and experimental setup, Section 3 provides the details of the simulation of current and voltage pulses, and Section 4 presents the results and discussions. Finally, in Section 5, we conclude.

\section{Discharge Circuit Characteristics and Experimental Setup}

The discharge circuit is shown in Figure 1 which had storage capacitor, $C_{s}=1.65 \mathrm{nF}$, charging through dc power supply and the inductance of $L_{c}=0.15 \mathrm{mH}$. $C_{p}=0.68 \mathrm{nF}$ is sharpening capacitor and Thy is the thyratron switch model TGI1-1000/25. Here, the inductance of the thyratron equivalent circuit is shown by $L_{1}$ and the inductance of the laser tube equivalent circuit is indicated by $L_{2}$.

The shape of the voltage and current waves would be recorded in the oscilloscope by use of a high voltage probe and a current Rogowski coil, which are illustrated in Figure 2.

\section{Simulation of Current and Voltage Pulses}

The differential equations describing the CVL circuit are as follows [31]:

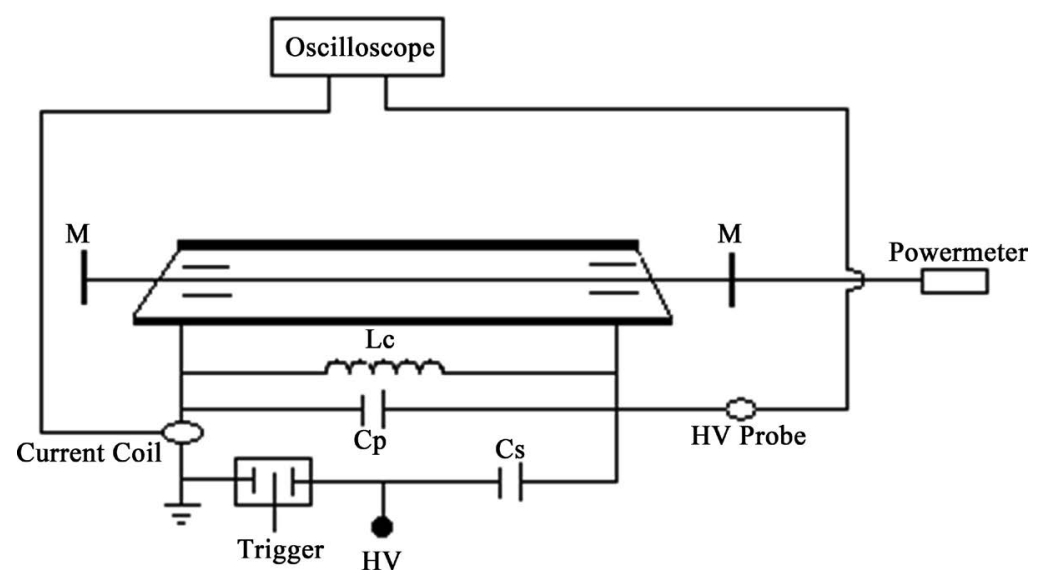

Figure 1. The schematic of experimental setup.

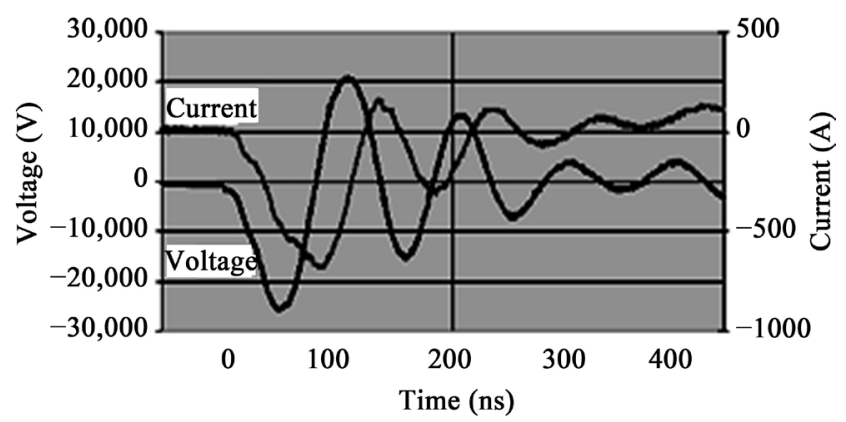

Figure 2. The shapes of the voltage and current waves of a CVL discharge tube. 


$$
\begin{aligned}
& \frac{\mathrm{d} I_{1}}{\mathrm{~d} t}=v_{1}-v_{2}-\frac{I_{1} R_{t h}}{L_{1}} \\
& \frac{\mathrm{d} I_{2}}{\mathrm{~d} t}=v_{2}-\frac{I_{2} R_{d}}{L_{2}} \\
& \frac{\mathrm{d} v_{1}}{\mathrm{~d} t}=-\frac{I_{1}}{C_{S}} \\
& \frac{\mathrm{d} v_{2}}{\mathrm{~d} t}=I_{1}-\frac{I_{2}}{C_{P}}
\end{aligned}
$$

The currents of $I_{1}$ and $I_{2}$ are flowing via inductances of $L_{1}$ and $L_{2}$, respectively. $V_{1}$ and $V_{2}$ are the voltages of capacitors of $C_{s}$ and $C_{p}$, respectively. $R_{t h}$ is the resistance of thyratron and $R_{d}$ is the resistance of laser tube, i.e. plasma resistance of laser tube due to the collisions between conduction electrons and the components of plasma such as copper and neon atoms, which can be obtained via following relation [31]:

$$
R_{d}=\frac{L m_{e} v_{m}}{a n_{e} e^{2}}
$$

where, $n_{e}$ is electron density, $e$ and $m_{e}$ are charge and mass of electron, respectively, and $v_{m}$ represents the collision frequency of momentum transmission. $L$ and $a$ are the length and cross section area of discharge tube, respectively. The collision frequency of $v_{m}$ is obtained by following relation:

$$
v_{m}=\overline{V_{e}}\left(N_{N e} \tilde{\sigma}_{e N e}+N_{C u} \tilde{\sigma}_{e c u}\right)
$$

where $N_{N e}$ and $N_{C u}$ are the density of neon and copper atoms, $\tilde{\sigma}_{e N e}$ and $\tilde{\sigma}_{e c u}$ are the cross sections of momentum transmissions due to neon and copper atoms, respectively. Generally, the momentum transmission cross sections of neon and copper atoms are $1.5 \times 10^{-20} \mathrm{~m}^{2}$ and $6 \times 10^{-20} \mathrm{~m}^{2}$, respectively. Usual density of copper atoms is $8 \times 10^{20} \mathrm{~m}^{-3}$ at neon buffer gas pressure of 35 torr, and the density of neon atoms can be obtained via following equation:

$$
N_{N e}=2.145 \times 10^{23} \frac{273}{T_{g}} p\left(\mathrm{~m}^{-3}\right)
$$

where $p$ is pressure of the buffer gas in torr and $T_{g}$ is the gas temperature in Kelvin. In Equation (7), these values were supposed for pressure and temperature of $p=35$ torr and $T_{g}=1800 \mathrm{~K}$. Therefore the resistance of plasma in laser tube could be obtained by the following relation:

$$
R_{d}=3032.2\left(\frac{3}{2} K T_{e}\right)^{\frac{1}{2}}
$$

where (3/2)KT $T_{e}$ is the equivalent energy of electron temperature in $\mathrm{eV}$. In the condition of optimized performance of CVL, this value would be $7 \mathrm{eV}$.

The resistance of thyratron is equal to:

$$
R_{t h}=10^{7} \exp \left(-t / \tau_{S}\right)+10^{-2}
$$

where $\tau_{s}$ represents time coefficient of thyratron and equals to $20 \mathrm{~ns}$. In this simulation, the values of elements are as follow: $L_{1}=2 \mathrm{nH}, L_{2}=4 \mathrm{nH}, C_{s}=3 \mathrm{nF}$ and $C_{p}=1 \mathrm{nF}$. By solving the Equations (1)-(9) by Runge-Kutta method in Matlab software, the behavior of current-voltage plot of discharge tube would be obtained which is depicted in Figure 3.

\section{Results and Discussions}

By comparison of Figure 2 and Figure 3 it can be find that the simulated behavior of current-voltage is very close to the experimental results. In both of the plots, the phase retard between current and voltage is observable which could be attributed to the existence of impedances. The oscillations of current and voltage waves show 


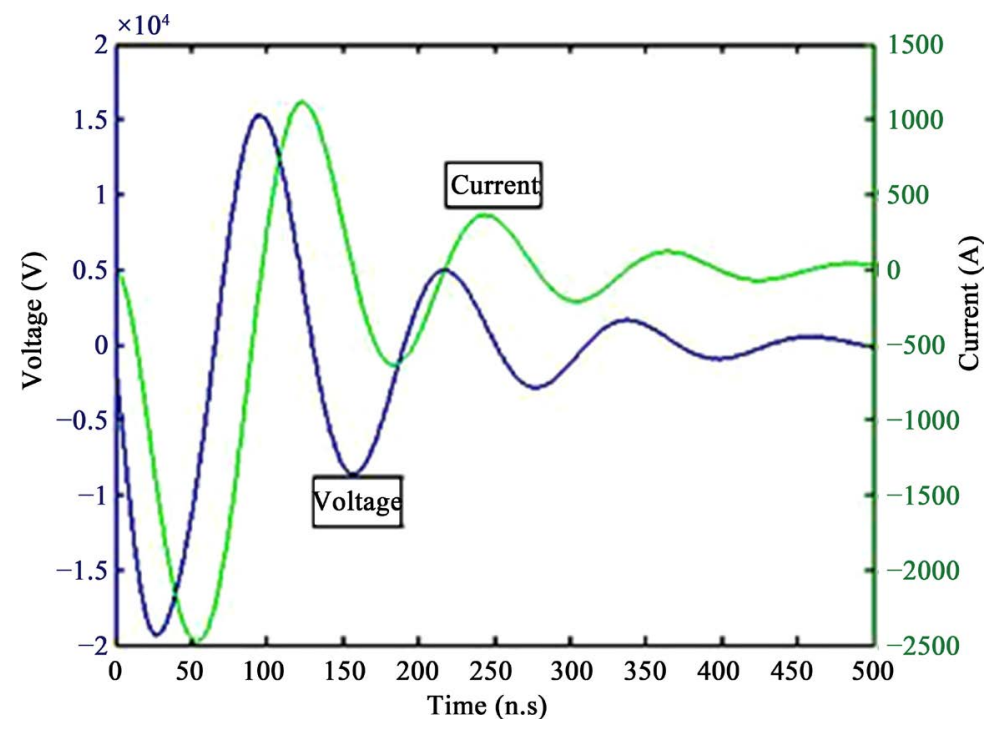

Figure 3. The simulation of current and voltage pulses of discharge.

that the circuit impedance is higher than that of laser tube, which is a common issue in the gas lasers. The laser tube is a non-linear load whose impedance depends on the geometry characteristics and impedance (capacity and inductance of tube) and conductivity of plasma. Each of these parameters has time dependency on rising time and the strength of longitudinal electric field in laser; also the shape of the current pulse depends on impedance of the tube. Therefore the matching of the impedance of external circuit with that of laser tube is crucial. Generally the higher current rates cause the higher inversion population, which the gain and output power of laser would be increased. When the optimum of current raise time is established, by increasing of applied voltage on electrodes tube and partial pressure of copper vapor, finally the output power of laser would be saturated due to more ionization and fast discharge of ground state of copper atoms. If the pressure of copper vapor is increased, the voltage of discharge tube could be increased, to reach higher output power. However, there are some limitations for application of high voltage on laser tubes' ends. In order to operate in high pressure of copper vapor and required high voltages for specific high power outputs, the voltage pulse should be applied in shorter time than the breakdown time characteristic of laser tube. This could be achieved by reduction of tube's inductance and application of compressed magnetic pulses, so. However for a CVL, the current rate should not be increased very fast. Otherwise, high rate of ionized copper atoms leads to reduction of populated desired levels and the period of gain would be shortened due to discharge of ground state.

Therefore by simulation through different retard phases between discharge current and voltage pulses, and also the raising time of current and consideration of their optimized values to obtaining the maximum output power, it is possible to estimate the numerical values of elements (capacity of capacitors, and inductances) before operation of an experiment, and then apply the optimum values for practical purposes.

\section{Conclusion}

The present developments of copper vapor laser technology were computational modeling and simulations methods including the discharge circuitry. Generally, the electrical parameters can be predicted using the physical simulation codes before the experiment. The current and voltage pulses of the discharge tube were investigated by solving the equations of circuit via Runge-Kutta method. The results showed close agreement between the modeling and the experimental results.

\section{References}

[1] Kharasani, K., Behrouzinia, S. and Salehinia, D. (2008) Air as a Buffer Gas in Metal-Vapor Lasers. Journal of Russian Laser Research, 29, 599-603. http://dx.doi.org/10.1007/s10946-008-9047-9

[2] Kharasani, K., Salehinia, D., Behrouzinia, S., Sajad, B. and Parvizian, M. (2008) Frequency Dependence of the Output Power of Metal Vapor Lasers. Optics Communications, 281, 3799-3801. 
http://dx.doi.org/10.1016/j.optcom.2008.03.059

[3] Zoghi, M., Parvin, P., Behrouzinia, S., Salehinia, D., Kharasani, K. and Mehravaran, H. (2009) Acoustic Effects of Metal-Vapor Lasers. Applied Optics, 48, 3460. http://dx.doi.org/10.1364/AO.48.003460

[4] Faez, R., Marjani, A. and Marjani, S. (2011) Design and Simulation of a High Power Single Mode $1550 \mathrm{~nm}$ InGaAsP VCSELs. IEICE Electronics Express, 8, 1096-1101. http://dx.doi.org/10.1587/elex.8.1096

[5] Marjani, S., Faez, R. and Marjani, H. (2011) An Impact of the Hole Etching Depth within a Photonic Crystal VCSEL on Its Heat Sources. Australian Journal of Basic and Applied Sciences, 5, 766-770.

[6] Marjani, S., Faez, R. and Marjan, A. (2011) Design and Modeling of a High Single Mode Power Long Wavelength InGaAsP Photonic Crystal VCSEL. Australian Journal of Basic and Applied Sciences, 5, 1064-1069.

[7] Marjani, A., Marjani, S. and Shirazian, S. (2011) Numerical Simulation of Silicon Carbide Polymers (6H-SiC \& 3C-SiC) as the Active Area for $0.83 \mu \mathrm{m}$ Wavelength Semiconductor Laser. The 14th Iranian Physical Chemistry Conference, University of Tehran, Kish, 876-878.

[8] Marjani, S., Faez, R. and Marjani, H. (2011) Analysis and Design of Semiconductor Laser with Silicon Carbide Polymers (6H-SiC and 3C-SiC). Australian Journal of Basic and Applied Sciences, 5, 1060-1063.

[9] Marjani, S., Rahnama, M. and Marjani, H. (2011) Numerical Optimization of Single-Mode InGaAsP Vertical-Cavity Surface-Emitting Lasers. Australian Journal of Basic and Applied Sciences, 5, 1207-1211.

[10] Marjani, S. and Marjani, H. (2011) Effects of Lattice Temperature on the Various Elements of Heat Sources in a Long Wavelength InGaAsP Photonic Crystal VCSEL. Australian Journal of Basic and Applied Sciences, 5, 1257-1261.

[11] Marjani, S. and Marjani, H. (2011) Analysis of Lattice Temperature Effects on a Long Wavelength InGaAsP Photonic Crystal VCSEL. Australian Journal of Basic and Applied Sciences, 5, 1374-1378.

[12] Marjani, S. and Marjani, H. (2012) Optimization of a Long Wavelength Vertical-Cavity Surface-Emitting Lasers by Employing Photonic Crystal. Asian Journal of Chemistry, 24, 3174-3176.

[13] Marjani, S. and Marjani, H. (2012) Self-Heating Effects in a Silicon Carbide Polymers (6H-SiC and 3C-SiC) Semiconductor Laser. Asian Journal of Chemistry, 24, 3145-3147.

[14] Marjani, S. and Marjani, H. (2012) Effects of Hole Etching Depth in a Long Wavelength InGaAsP Photonic Crystal Vertical Cavity Surface Emitting Laser. Asian Journal of Chemistry, 24, 3194-3196.

[15] Behrouzinia, S., Aghababaeinezhad, M., Khorasani, K., Sajad, B., Salehinia, D., Mahmoodabadi, Z.D. and Sabaghi, M. (2012) The Behaviour of Inverse Voltage on Thyratron's Anode versus Operational Parameters in Gold Vapour Laser. International Journal of Fundamental Physical Sciences, 2, 6-8. http://dx.doi.org/10.14331/ijfps.2013.330025

[16] Marjani, S. and Marjani, H. (2012) Effects of Lattice Temperature on the Various Elements of Heat Sources in Silicon Carbide Polymers (6H-SiC and 3C-SiC) Semiconductor Laser. Asian Journal of Chemistry, 24, 3123-3125.

[17] Marjani, S., Faez, R. and Marjani, H. (2012) Analysis of the Various Elements of Heat Sources in Silicon Carbide Polymers (6H-SiC and 3C-SiC) Semiconductor Laser. Asian Journal of Chemistry, 24, 2333-2335.

[18] Marjani, S., Faez, R. and Marjani, H. (2012) Design and Modeling of a Semiconductor Laser by Employing Silicon Carbide Polymers (6H-SiC, 3C-SiC and 4H-SiC). Asian Journal of Chemistry, 24, 2177-2179.

[19] Marjani, S., Faez, R. and Hosseini, S.E. (2013) Analysis of Lattice Temperature Effects on a GaInP/6H-SiC Strained Quantum-Well Lasers. Asian Journal of Chemistry, 25, 4715-4717. http://dx.doi.org/10.14233/ajchem.2013.13072

[20] Madadi, R., Marjani, S. and Faez, R. (2013) Silicon Carbide Polymers (6H-SiC, 3C-SiC and 4H-SiC) Semiconductor Laser: Influence of Self-Heating. Proceedings of the 3rd Iranian Conference on Optics and Laser Engineering (ICOLE), Isfahan, 9-10 October 2013, 1069-1072.

[21] Marjani, S. (2013) Various Elements of Heat Sources within an Optimized Photonic Crystal Vertical Cavity Surface Emitting Laser: Influence of Hole Etching Depth. Asian Journal of Chemistry, 25, 4153-4156. http://dx.doi.org/10.14233/ajchem.2013.13281

[22] Marjani, S. (2013) Optimization of an InGaAsP Vertical-Cavity Surface-Emitting Diode Lasers for High-Power Single-Mode Operation in 1550 nm Optical-Fibre Communication Systems. Asian Journal of Chemistry, 25, 4150-4152. http://dx.doi.org/10.14233/ajchem.2013.13186

[23] Marjani, S., Faez, R. and Hosseini, S.E. (2013) Threshold Characteristics Analysis of InP-Based PhC VCSEL with Buried Tunnel Junction. Proceedings of the 21st Iranian Conference on Electrical Engineering (ICEE), Mashhad, 14-16 May 2013, 1-4. http://dx.doi.org/10.1109/iraniancee.2013.6599783

[24] Majdabadi, A., Marjani, S. and Sabaghi, M. (2014) Threshold Characteristics Enhancement of a Single Mode $1.55 \mu \mathrm{m}$ InGaAsP Photonic Crystal VCSEL for Optical Communication Systems. Optics and Photonics Journal, 4, 296-303. http://dx.doi.org/10.4236/opj.2014.410029

[25] Naeemi, M.A., Marjani, S. and Peiravi, A. (2014) Time to Failure Analysis of Single Mode Long-Wavelength In- 
GaAsP Vertical-Cavity Surface-Emitting Lasers. Proceedings of the 22nd Iranian Conference on Electrical Engineering (ICEE), Tehran, 20-22 May 2014, 43-47. http://dx.doi.org/10.1109/iraniancee.2014.6999500

[26] Behrouzinia, S., Sadighi, R. and Parvin, P. (2003) Pressure Dependence of Small-Signal Gain and Saturation Intensity of a Copper Vapor Laser. Applied Optics, 42, 1013. http://dx.doi.org/10.1364/AO.42.001013

[27] Behrouzinia, S., Sadighi, R. and Parvin, P. (2004) Temperature Dependence of the Amplifying Parameters of a Copper Vapor Laser. Laser Physics, 14, 1050-1053.

[28] Little, C.E. (1999) Metal Vapor Lasers. John Wiley \& Sons, Chichester.

[29] Carman, R.J., Brown, D.J.N. and Piper, J.A. (1994) A Self-Consistent Model for the Discharge Kinetics in a HighRepetition-Rate Copper Vapor Laser. IEEE Journal of Quantum Electronics, 30, 1876-1895. http://dx.doi.org/10.1109/3.301652

[30] Kashner, M.J. (1983) Laser-Bore Copper-Vapor Laser: Kinetics and Scaling Issues. Journal of Applied Physics, 54, 2970. http://dx.doi.org/10.1063/1.332499

[31] Deli, Y. (2004) Numerical and Experimental Analysis of Middle-Bore Copper-Vapor Laser Discharge. Proceedings of the SPIE 3549, High-Power Lasers: Solid State, Gas, Excimer, and Other Advanced Lasers II, 14, 144. http://dx.doi.org/10.1117/12.344114 